\title{
Messrechnen: 350 Jahre Rechenschieber
}

\begin{abstract}
Heinz Joss
Heinz Joss, geboren 1929, studierte Architektur an der ETH in Zürich. Seinen Beruf übte er längere Zeit in Schweden aus, bevor er 1961 in Zürich Aufbau und Leitung einer Forschungs- und Entwicklungsstelle der schweizerischen Bauwirtschaft übernahm. Während der letzten zehn Jahre seiner beruflichen Tätigkeit widmete er sich der Nahtstelle zwischen Sprache und Bauen. Vor einigen Jahren begann er, analoge logarithmische Rechengeräte zu sammeln, und heute ist er Besitzer einer umfangreichen Sammlung von Rechenstäben, -scheiben und -walzen.
\end{abstract}

Man vergisst im menschlichen Leben nichts so leicht als das Multiplizieren, wenn man es noch so gut in der Schule gelernt hat und kann.

\author{
Johann Peter Hebel (1760-1826) \\ im Schatzkästlein des rheinischen Hausfreundes
}

\section{Eine Reihe von Entdeckungen und Erfindungen}

Der schottische Mathematiker John Napier (1550 bis 1617), oft latinisiert Neper genannt, berechnete in zwanzigjähriger Arbeit die von ihm entdeckten Logarithmen und publizierte sie 1614 in Mirifici logarithmorum canonis descriptio (Beschreibung der wunderbaren Gesetzmässigkeit der Logarithmen). Dass der Schweizer Jost Bürgi nach

Ältere erinnern sich noch: Bevor Taschenrechner existierten, war der Rechenschieber der ständige Begleiter des Technikers und Ingenieurs und wohl auch ein Statussymbol dieses Berufstandes. Dazu lässt sich Robert Musil zitieren; als ausgebildeter Maschineningenieur und ehemaliger Student der Mathematik wusste Musil, wovon er sprach. In seinem umfangreichen, unvollendet gebliebenen Werk Der Mann ohne Eigenschaften sagt er: Der Rechenschieber, das sind zwei unerhört scharfsinnig verflochtene Systeme von Zahlen und Strichen; der Rechenschieber, das sind zwei weiss lackierte, ineinander gleitende Stäbchen von flach trapezförmigem Querschnitt, mit deren Hilfe man die verwickeltsten Aufgaben im Nu lösen kann, ohne einen Gedanken nutzlos zu verlieren; der Rechenschieber, das ist ein Symbol, das man in der Brusttasche trägt und als einen harten weissen Strich über dem Herzen fühlt. - Etwas zur Geschichte dieses so ausserordentlich erfolgreichen Rechengerätes erzählt uns Heinz Joss in seinem Beitrag. ust 
heutigen Kenntnissen die Logarithmen schon vorher entdeckt hatte, steht damit in keinem Zusammenhang, da zu jenem Zeitpunkt Bürgis Entdeckung noch nicht bekannt war; Bürgi veröffentlichte sie nämlich erst 1620. Der englische Professor für Geometrie, Henry Briggs (1561 bis 1630), berechnete später die dekadischen Logarithmen und brachte sie 1617 unter dem Titel Logarithmorum chilias prima (Der Logarithmen erstes Tausend) heraus.

Sein Landsmann Edmund Gunter (1581 bis 1626), Pfarrer, Mathematiker und Astronom, hatte als erster die Idee, Briggs' Logarithmen graphisch darzustellen, indem er sie als Skala auf einer 2 Fuss langen hölzernen Zeichenschiene auftrug. Er publizierte seine Erfindung 1623 in De Sectore \& Radio (Über die Sektoren und den Radius). Die Gunter's Scale erlaubte erstmals graphisches logarithmisches Rechnen: Mit Hilfe zweier Stechzirkel wurden die benötigten Zahlen auf den Skalen abgegriffen. Der Gunter-Stab erlangte schnell grosse Bedeutung. Versehen mit einer logarithmischen und ergänzt mit trigonometrischen Skalen diente er astronomischen, navigatorischen und auch ballistischen Zwecken. So soll Admiral Nelson 1805 in der Schlacht von Trafalgar die Gunter's Scale zum Lösen nautisch-taktischer Probleme erfolgreich eingesetzt haben. Der Gunter-Stab war zwar noch kein Rechenschieber, aber er gilt doch als dessen unmittelbarer Vorläufer; er blieb zudem in der Schiffahrt bis Ende des letzten Jahrhunderts, also noch 170 Jahre nach der Erfindung des Rechenschiebers, in Anwendung, oft in einer ergänzten Ausführung Navigation Scale, improved by B. Donn.

Der Engländer William Oughtred (1575 bis 1660), Pfarrer und später Professor für Mathematik, gilt als Erfinder des Rechenschiebers. Um das Jahr 1630 hat er die Erfindung Gunters benützt, um aus ihr den Rechenschieber zu entwickeln, der dann bis in die 1970er-Jahre das tägliche Werkzeug aller darstellte, die beruflich zu rechnen hatten. Oughtred hatte nämlich die Idee, die von Gunter eingeführten Skalen je an der Kante zweier Stäbe aufzutragen, die, gegeneinander verschiebbar, den ersten wirklichen Rechenschieber darstellten; die bei der Gunter's Scale noch erforderlichen zwei Stechzirkel erübrigten sich nun. Mit Gunter und Oughtred war die Idee des Messrechnens geboren. (Der Begriff Messrechnen wurde von Heinrich Daemen-Schmid um 1920 in der Werbung für seine Loga-Rechenwalzen verwendet; er charakterisiert die Technik des Schieberrechnens hervorragend.)

\section{Die Genauigkeit des Schieberrechnens}

Analoges Rechnen stösst bezüglich Genauigkeit an Grenzen der Gerätekonstruktion, der Einstellung und der Ablesung. In einer Studie hat Prof. Karl Strubecker 1966 die relative Genauigkeit des Rechenschiebers mit 0,5\%o angegeben. Für das praktische Rechnen war dies in den weitaus meisten Fällen ausreichend, so dass der Rechenschieber während 350 Jahren das verbreitetste Rechengerät darstellte.

Die Erhöhung der absoluten Genauigkeit bildete aber doch das Ziel vieler Verbesserungsbestrebungen: Das Erreichen einer grösseren Skalenlänge durch längere Rechenstäbe, Verschränken der Skalen, kreisförmige oder gar zylindrische Rechenschieber war der eine Ansatz. Während der Standardrechenstab eine Skalenlänge von $25 \mathrm{~cm}$ aufwies, wurden auch Stäbe mit $50 \mathrm{~cm}$, Scheiben mit $75 \mathrm{~cm}$ und Walzen mit bis zu $24 \mathrm{~m}$ Skalenlänge hergestellt. Ein anderer Ansatz war das Anbringen von vergrössernden Läufern oder gar von Läufern mit einer Stativlupe. Verbesserte Genauigkeit, ob tatsächlich 
erreicht oder nur behauptet, bildete denn auch immer wieder ein Werbeargument für verschiedene Marken oder Typen. Ein anerkannter Praktiker des Rechenschiebers hat sich jedoch zum Thema der Genauigkeit sehr kategorisch geäussert: Derjenige, für den die Genauigkeits- und Stellenfrage ein Problem ist, der hat das Wesen des Rechenschiebers überhaupt nicht erfasst (M. Hartmuth, 1942 in Vom Abakus zum Rechenschieber).

\section{Die unterschiedlichen Formen des Rechenschiebers}

Bereits Oughtred sah die Möglichkeit, die Skalen sowohl gerade als auch kreisförmig zu gestalten und legte seine Erfindung in beiden Formen vor. Dass in der Folge die Grosszahl der Rechenschieber in der Form der Rechenstäbe mit geraden Skalen gebaut wurde, hatte vermutlich, wenigstens in den früheren Zeiten, herstellungstechnische Gründe. Der Rechenstab mit seinem zweiteiligen Körper und einer dazwischen laufenden verschiebbaren Zunge blieb bis zum Ende der Rechenschieberära um 1975 die vorherrschende Form des Rechenschiebers. Diese Bauweise gestattete ursprünglich nur das Anbringen von zwei Skalenpaaren, je an der hinteren bzw. vorderen Berührungskante von Körper und Zunge. Weil man sich sehr bald nicht mehr mit zwei Skalenpaaren begnügen mochte, wurden Rechenstäbe mit zwei, drei oder gar vier Zungen gebaut, es wurde mit einer wendbaren Zunge oder mit mehreren auswechselbaren Zungen gearbeitet. Die Beziehungen zwischen den Skalen der verschiedenen Zungen herzustellen, blieb jedoch bei solchen Konstruktionen dem Intellekt des Anwenders überlassen. Erst der Läufer, der mit seinem Indexstrich gestattete, auch sich nicht direkt berührende Skalen miteinander in Verbindung zu bringen, ermöglichte es, auf einem Rechenstab mit nur einer Zunge eine grössere Anzahl von Skalen so unterzubringen, dass der Übergang von einer Skala $\mathrm{zu}$ einer anderen direkt erfolgen konnte.

Der Rechenstab als die verbreitetste Form des Rechenschiebers hatte nicht nur Vorteile. Neben der aus Gründen der Handhabbarkeit beschränkten Skalenlänge (d.h. relativ geringe Genauigkeit) war es vor allem das sogennante Durchschieben, das als Nachteil empfunden wurde. Unter Durchschieben versteht man das Verschieben der Zunge um die gesamte Skalenlänge nach links bzw. nach rechts, wenn bei bestimmten Zahlenkombinationen das Resultat auf der Zungenskala ausserhalb der Körperskala zu stehen kommt. Um diesen Nachteilen zu begegnen, wurden andere Formen des Rechenschiebers entwickelt.

Die Rechenscheibe mit kreisförmiger, seltener mit spiralförmiger Anordnung der Skalen hat sich erst viel später einigermassen durchzusetzen vermocht, als die herstellungsbedingten Probleme technisch zu bewältigen waren. Die Scheibe weist, bei einem der Stablänge entsprechenden Durchmesser, eine grössere Skalenlänge auf und kommt ohne das Durchschieben aus (Werbeslogan immer vorwärts rechnen). Rechenscheiben bildeten zeitweise eine beliebte Alternative zum Rechenstab, wenn auch ihre Produktion nie die Stückzahlen der Rechenstäbe zu erreichen vermochte. - Eine Spielart der Rechenscheibe bilden die Rechenuhren, bei denen die Rechenscheibe in ein Gehäuse in der Form einer Taschenuhr eingebaut ist; die Skalen und die Indexlinie werden dabei über eine oder zwei Kronen angetrieben.

Von Rechenschiebern in der Form eines Rechenrings ist bisher nur ein einziges Exemplar bekannt; vier Skalen und ein Index sind auf drei parallelen, gegeneinander verdrehbaren 
Zylindermänteln angebracht. Dasselbe Prinzip wurde auch bei Rechenrädern angewendet, bei denen aber die wesentlich grösseren Zylindermäntel mit Speichen auf ihre Drehachse abgestützt sind. Von ihnen sind noch mehrere Stücke erhalten.

Da wie bereits erwähnt, die Genauigkeit eines Rechenschiebers u.a. eine Funktion seiner Skalenlänge ist, wurde immer wieder versucht, längere Skalen zu verwenden, ohne die Handlichkeit des Geräts zu beeinträchtigen. Auf dem Umweg über den Rechenrost, der auf der Aufteilung eines sehr langen Rechenstabes in Einzelabschnitte und deren paralleler Anordnung in Form eines Gitterrostes beruhte, stellte erst die Anbringung dieser Einzelabschnitte auf einem Zylindermantel eine praktikable Lösung dar; solche Rechenwalzen wurden in der Folge mit Skalenlängen bis zu 24 m gebaut und genossen während längerer Zeit eine beachtliche Verbreitung.

Eine interessante Variante der Rechenwalzen bildeten die englischen Rechenzylinder, bei denen die Skala in Form einer Schraubenlinie mit kleinem Steigungswinkel aufgebracht ist, was auch bei kleinen Abmessungen des Geräts eine grosse Skalenlänge ermöglichte.

\section{Die Skalensysteme}

Der spätere französische Artillerieoberst und Professor für Mathematik Amédée Mannheim (1831 bis 1906) hat um 1850 eine Skalenkombination und -anordnung entwickelt, die erstmals grosse und herstellerunabhängige Verbreitung erfuhr. Die Vorderseite wies die Grundzahlen- und die Quadratskalenpaare auf, die Rückseite der Zunge eine Sinusund eine Tangensskala; für Berechnungen mit diesen beiden Skalen musste die Zunge gewendet werden. Das System Mannheim bildete gewissermassen einen ersten internationalen Standard und damit auch die Grundlage für die damalige und spätere industrielle Rechenstabproduktion. Der Läufer, der es gestattete, die Anwendung mehrerer Skalen miteinander zu verbinden, war zwar schon 1770 vom Engländer John Robertson erfunden worden, fand aber erst mit dem System Mannheim allgemeine Verbreitung.

Der deutsche Ingenieur Max Rietz (1872 bis 1956) ergänzte 1902 die Mannheimschen Skalen mit einer Kuben- und einer Mantissenskala; Indexlinien auf der Körperrückseite gestatteten, die Sinus- und Tangensskalen ohne Umdrehen der Zunge anzuwenden. Das System Rietz war bis zum Ende der Rechenschieberproduktion eine der meistangewendeten Skalenanordnungen. Sie hat das System Mannheim völlig verdrängt.

1934 wurde das System Rietz nochmals weiterentwickelt und ergänzt. An der Technischen Hochschule Darmstadt wurde eine neue Skalenauswahl und -anordnung erarbeitet; gegenüber dem System Rietz war die Vorderseite mit einer Pythagoräischen Skala $\sqrt{1-x^{2}}$ ergänzt, die Mantissenskala auf die hintere Längskante verschoben, die Sinusund Tangensskalen auf die vordere Längskante, so dass die Zungenrückseite für drei Exponentialskalen frei wurde. Diese Verbesserung kam den Bedürfnissen des Ingenieurs entgegen; dem Vater des Systems Darmstadt, Prof. Alwin Walther (1898 bis 1967), sollen deshalb seine Mathematikerkollegen vorgeworfen haben, die Mathematik an die Ingenieure verraten zu haben. Das System setzte sich aber durch und blieb bis zum Ende der Rechenschieberära neben dem System Rietz im Angebot der Hersteller.

Später wurde mit der Einführung des doppelseitigen sog. Duplex-Rechenstabs das Anfügen weiterer Skalen möglich (vgl. Abbildungen). 

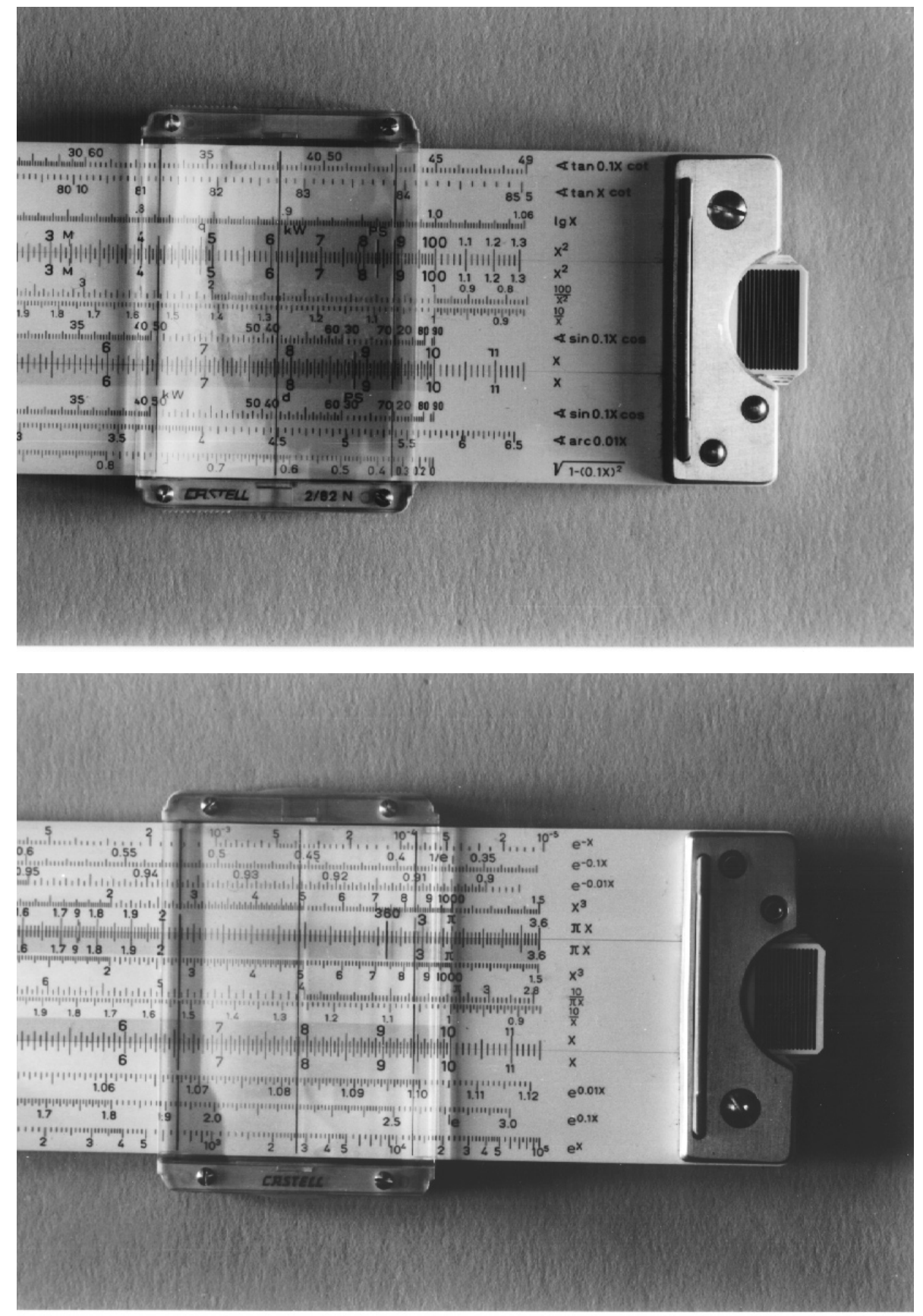

Faber-Castell Novo-Duplex 2/82 N mit erweitertem Skalenbild, gebaut 1972, einer der vollständigsten und komplexesten Rechenschieber der allerletzten Ära. Oben: Rechtes Ende der Vorderseite, unten: Rechtes Ende der Rückseite. 


\section{Länder und Hersteller}

Nach Erfindung des Rechenstabes nahm sich in England eine Vielzahl von Instrumentenmachern des Baus von Rechenschiebern an. Buchsbaumholz und Messing waren die Hauptwerkstoffe; bei teureren Ausführungen trat Elfenbein an die Stelle des Holzes, der höhere Kontrast zur schwarzen Gravur verbesserte die Lesbarkeit. Neben den rein mathematischen Skalen gab es sehr viele anwendungsbezogene Spezialskalen. Es war die Epoche der Einzelanfertigungen und der Kleinstserien. - Ausserhalb Englands waren Rechenschieber bis zum Beginn des 19. Jahrhunderts kaum bekannt.

Nach dieser Periode der englischen Dominanz folgte eine französische (ca. 1800 bis 1880). Die Firmen Lenoir und später Tavernier-Gravet erlangten mit ihren Produkten Weltbedeutung. Auch der bereits erwähnte Entwicklungsbeitrag Mannheims dürfte zum Ansehen der französischen Rechenschieber wesentlich beigetragen haben.

Eine Importsperre während des deutsch-französischen Krieges gab der deutschen Industrie den Impuls, die eigene Rechenschieberherstellung zu wagen (ab 1870). Die drei grossen Hersteller Dennert \& Pape (ab 1936 mit der Marke Aristo), Faber-Castell und Nestler, aber auch andere, gehörten in der Folge weltweit zu den dominierenden Produzenten. Dennert \& Pape entwickelte gegen 1890 die dann für Jahrzehnte vorherrschende Bauweise des Holzstabes mit Celluloidauflage; als Unterlage wurden die unterschiedlichsten Hölzer verwendet. Diese Bauweise wurde ab 1936 nur sehr langsam durch die Vollkunststoff-Rechenstäbe verdrängt.

$\mathrm{Zu}$ diesen drei Firmen mit Weltruf gesellten sich dann die drei amerikanischen Firmen Dietzgen, Keuffel \& Esser und Picket \& Eckel sowie die japanische Firma Hemmi mit der Marke Sun. Die Japaner bauten als einzige Rechenstäbe aus Bambus, einem dazu ganz hervorragend geeigneten Material.

In der Schweiz waren es die drei Hersteller Billeter, Loga und National, die internationale Bedeutung erlangten. Bei ihnen stellten die Rechenwalzen und später die Rechenscheiben Schwerpunkte der Produktion dar. Offenbar vermochten sie mit ihnen eine Marktnische zu besetzen und sich dadurch gegen die grossen ausländischen Hersteller von Rechenstäben zu behaupten. Allein Loga soll nach eigenen Angaben Zehntausende von Rechenwalzen hergestellt und in alle Welt verkauft haben.

\section{Quellen}

- Deutsches Museum, München: Ausstellung

- Jezierski, Dieter von: Rechenschieber, eine Dokumentation, Selbstverlag des Autors, Stein b. Nürnberg, 1997

- Journal of the Oughtred Society, Palo Alto, California, USA.

- Museum of Science, London: Ausstellung

- Technorama, Winterthur: Ausstellung, Archiv und Lager

- Whipple Museum of the History of Science, Cambridge: Ausstellung

Heinz Joss

dipl. Architekt ETH/SIA

Rainring 4

CH-8108 Dällikon 